\title{
Niveau de consommation en acides gras polyinsaturés de la femme allaitante en France: étude de consommation INCA2 et évolution du contenu en acides gras essentiels du lait maternel de 1997 à 2014
}

Carole Vaysse $^{2, *}$, Noémie Simon ${ }^{1, *}$, Jessica Tressou ${ }^{3}$, Stéphane Pasteau $^{4}$, Benjamin Buaud ${ }^{2}$, Philippe Guesnet ${ }^{5}$, Leslie Couedelo ${ }^{2}$ et Claude Billeaud ${ }^{6}$

1 Terres Univia, Paris, France

2 ITERG, Canéjan, France

3 INRA, Paris, France

${ }^{4}$ Phasme Consulting, Paris, France

${ }^{5}$ PG Consulting, Bures sur Yvette, France

${ }^{6}$ CIC Pédiatrique 1401, CHU Pellegrin, Bordeaux, France

Reçu le 25 avril 2018 - Accepté le 29 avril 2018

\begin{abstract}
Résumé - Les données de consommation en acides gras polyinsaturés (AGPI) de la femme allaitante sont rares bien qu'essentielles pour évaluer le niveau d'apport en acides gras du nourrisson et éventuellement orienter la mère vers une alimentation adaptée voire vers une supplémentation en acides gras essentiels. Les données INCA 2 concernant les femmes allaitantes ont été combinées aux données de composition nutritionnelle des aliments de la base de données Ciqual 2013 pour évaluer leurs apports en acides gras. Bien qu'il s'agisse d'un échantillon limité d'individus enquêtés il y a 12 ans, ces données restent malgré tout intéressantes à analyser et à comparer aux recommandations actuelles de l'Anses. Elles montrent que la consommation en AGPI n-3 n'est pas suffisante au regard des recommandations. Par ailleurs, il a été démontré que la composition en acides gras du lait maternel est un marqueur reconnu de la consommation des lipides alimentaires et un bon reflet du niveau de consommation des AGPI n-6 et n-3. Or, les données épidémiologiques relatives à la quantité et à la qualité des lipides consommés en France sont rares. Dans le cadre de différentes études cliniques conduites sur le lait maternel, l'ITERG a pu ainsi estimer l'évolution des habitudes alimentaires des Français depuis près de 17 ans. Un travail de synthèse a repris l'ensemble des données acquises au cours de 4 études cliniques menées entre 1997 et 2014. Cette synthèse a mis en évidence une évolution positive des proportions en AGPI n-3 en 17 ans (1997-2014). Toutefois, les proportions d'acide alpha-linolénique (ALA) et d'acide docosahexaénoïque (DHA) dans le lait maternel restent en deçà des recommandations nutritionnelles pour le nouveau-né.
\end{abstract}

Mots clés : apports en acides gras / lait maternel / nourrisson / recommandations

\begin{abstract}
Polyunsaturated fatty acids consumption in lactating women in France: the INCA2 study and evolution of essential fatty acids composition in breast milk from 1997 to 2014. Polyunsaturated fatty acid (PUFA) composition data of the breast milk are rare but essential for assessing the adequate intake of fatty acids in the newborn and possibly directing the mother to a suitable diet or even supplementation with essential fatty acids. The raw data for lactating women from the national study on individual food consumption 2006-2007 (INCA 2) were combined with the French food composition table (Ciqual 2013) to assess their fatty acid intakes. Although this is a limited number of subjects, these data are still interesting to analyze and compare with current Anses recommendations. According to these results, omega-3 PUFA
\end{abstract}

\footnotetext{
*Correspondances : n.simon@terresunivia.fr;

c.vaysse@iterg.com
} 
consumption is not sufficient for the purposes of the Recommended Dietary Intakes (RDI). In addition, it has been shown that the fatty acid composition of breast milk is a proven marker of dietary lipid consumption and a good reflection of the consumption's level of omega- 6 and omega-3 PUFAs. Epidemiological data on quantity and quality of lipids consumed in France are rare. In the context of different observational and interventional studies conducted on breast milk, ITERG has been able to estimate the evolution of French food habits for nearly 17 years. A synthesis work has taken over all the data acquired during 3 clinical studies carried out between 1997 and 2014. This synthesis showed a positive evolution of the proportions in omega-3 PUFA in 17 years (1997-2014). However, the proportions of alpha-linolenic acid (ALA) and docosahexaenoic acid (DHA) in breast milk remain below nutritional recommendations for the newborn.

Keywords: fatty acid intakes / breast milk / infant / recommendations

\section{Introduction}

Les acides gras polyinsaturés (AGPI) sont impliqués dans de multiples processus physiologiques et sont essentiels pour le développement et la santé de l'Homme. De nombreux travaux de recherche ont démontré le bénéfice d'un apport adéquat en AGPI dans la prévention de nombreuses maladies, en particulier des maladies cardiovasculaires, mais aussi sur l'hypertension, le diabète, la sensibilité à l'insuline et les troubles inflammatoires (Simopoulos, 2011). Ils sont également considérés comme potentiellement protecteurs vis-à-vis de certaines neuropathologies (Guesnet et al., 2005).

Il existe deux « familles » d'AGPI : les AGPI oméga-6 tels que l'acide linoléique (18:2n-6, LA) et l'acide arachidonique (20:4 n-6, AA) et les AGPI oméga-3, composés des acides alpha-linolénique (18:3n-3, ALA), eicosapentaénoïque (20:5n-3, EPA) et docosahexaénö̈que (22:6n-3, DHA).

Les deux précurseurs de chacune des deux familles (l'ALA pour les oméga-3 et le LA pour les oméga-6) doivent être apportés par l'alimentation, car l'Homme n'est pas capable de les synthétiser. L'apport en EPA et DHA est également nécessaire, la capacité de l'organisme à les synthétiser à partir de l'ALA n'étant pas suffisante pour répondre à ses besoins.

Des organismes internationaux et nationaux, comme l'Agence nationale de sécurité sanitaire de l'alimentation, de l'environnement et du travail (Anses) pour la France, ont proposé des apports alimentaires recommandés pour les AGPI pour la population adulte générale (Anses, 2011; Sioen et al., 2017). Cependant, la plupart des régimes alimentaires actuels dans le monde industrialisé occidental sont généralement pauvres en AGPI oméga-3, principalement sous la forme de chaînes longues (AGPI-LC) (EPA, DHA) (Sioen et al., 2017). Plus spécifiquement en France, les données de l'enquête nationale INCA 2 ont rapporté qu'au moins $85 \%$ de la population adulte ne répondaient pas aux recommandations d'apport pour l'EPA et le DHA, ainsi que pour leur précurseur essentiel (ALA) (Tressou et al., 2016).

Or, un faible apport alimentaire en AGPI oméga-3, notamment en DHA, pourrait avoir un impact plus défavorable sur la santé des nourrissons. Particulièrement abondant dans les lipides membranaires du cerveau et de la rétine, le DHA est un acide gras important pour la vision et le développement neurosensoriel du nouveau-né. Pour couvrir ses besoins en DHA mais aussi en AA, dont les contenus cérébraux augmentent jusqu'à l'âge de 2 ans, le nourrisson utilise les AGPI-LC présents dans le lait de sa mère ainsi que ceux qu'il synthétise à partir des précurseurs LA et ALA. Cependant, chez les enfants prématurés, ainsi que les enfants nés à terme, les quantités de DHA synthétisées apparaissent insuffisantes en regard des besoins. Sur le plan du développement neurosensoriel, le bénéfice d'une supplémentation en DHA a été clairement démontré (Uauy et al., 2003 ; Lauritzen et al., 2004). L'apport en AGPI du nouveauné dépend donc de la teneur en AGPI du lait maternel, qui lui-même dépend de l'alimentation de la mère (Jensen, 1999; Innis, 2004).

En effet, si le lait humain contient une quantité de lipides relativement constante, sa composition en acides gras peut varier de façon substantielle en fonction de l'alimentation de la mère (Read et al., 1965; Koletzko et al., 1992). Des études d'observation et d'intervention ont montré que les niveaux de LA, ALA, DHA ainsi que ceux de l'acide oléique et des acides gras trans mesurés dans le lait humain variaient avec les niveaux de ces acides gras dans l'alimentation maternelle, soulignant ainsi l'intérêt de ce marqueur biologique (Innis, 2007a) du statut nutritionnel en AGPI des mères.

Les données de consommation en AGPI de la femme allaitante sont rares bien qu'essentielles pour évaluer le niveau d'apport en acides gras du nourrisson et éventuellement orienter la mère vers une alimentation adaptée voire vers une supplémentation en acides gras essentiels pendant sa grossesse et son allaitement.

L'objectif de la présente étude était d'explorer chez les femmes allaitantes françaises les apports alimentaires en acides gras, en particulier les AGPI, en utilisant les données nationales les plus récentes sur la consommation alimentaire issues de l'étude INCA2 (Anses, 2009) et le contenu nutritionnel des aliments consommés issu des données Ciqual (Centre d'information français sur la qualité des aliments) (Anses, 2013).

Parallèlement, dans le cadre d'études cliniques conduites chez la femme allaitante depuis 1997 en collaboration avec le Dr Claude BILLEAUD (CIC pédiatrique 1401 du Centre Hospitalier Universitaire (CHU) de Bordeaux) et plusieurs lactariums de France, l'ITERG a pu suivre l'évolution de la composition en acides gras du lait maternel, et apprécier ainsi l'évolution sur une période de près de 20 ans de la consommation des AGPI en France. 


\section{2 Étude de consommation en acide gras polyinsaturés des femmes allaitantes}

\subsection{Méthodologie et analyse statistique des données INCA 2}

L'Anses a mis à disposition en accès libre, en 2013, une version détaillée de la table de composition nutritionnelle des aliments de la base de données Ciqual (Anses, 2013) et en septembre 2014, l'ensemble des données brutes obtenues dans INCA 2 (Anses, 2015). Afin d'évaluer l'évolution de la consommation en acides gras détaillée de la population française au regard des recommandations, Terres Univia a fait réaliser en 2014, une étude statistique détaillée des apports en acides gras de la population française. Ces apports ont pu être comparés aux apports nutritionnels conseillés (ANC) pour les acides gras définis par l'Anses en 2010 (Anses, 2011). Dans un deuxième temps, en 2017, le même travail a été réalisé sur des populations spécifiques: enfants et adolescents, femmes enceintes et allaitantes, séniors (Buaud et al., 2018). Seuls les principaux résultats obtenus pour les femmes allaitantes sont décrits dans cet article.

En suivant la même méthodologie que celle présentée dans l'article de Tressou et al. (2016), les registres alimentaires de 7 jours de l'enquête INCA 2 ont été combinés à la composition des aliments de la base de données Ciqual (Anses, 2013) pour calculer les apports en AGPI de 21 femmes allaitantes (2345 ans).

Malgré le souhait de rester au plus près des méthodes utilisées par l'Anses et ses groupes d'experts, étant donné l'échantillon limité d'individus dans le groupe de femmes allaitantes, ces données n'ont pas été pondérées.

La base de données Ciqual 2013 (Anses, 2013) répertorie la composition nutritionnelle de 1496 aliments pour 61 constituants. Pour cette analyse, les apports moyens journaliers en énergie, en lipides totaux et des principaux AGPI de la population de femmes allaitantes:

- l'apport énergétique total (AET) (kcal/jour);

- les lipides totaux (\% de l'apport énergétique sans alcool ou AESA);

- l'acide alpha-linolénique (ALA);

- l'acide linoléique (LA);

- les AGPI-LC: acide docosahexaénoïque (DHA) et acide eicosapentaénoïque (EPA).

À partir de ces derniers, ont été calculés :

- le rapport LA/ALA (ratio LA sur ALA);

- la somme EPA + DHA.

\subsection{Les besoins nutritionnels et recommandations en acides gras polyinsaturés chez la femme allaitante}

Les besoins nutritionnels physiologiques en AGPI de la femme allaitante (comme ceux de la femme enceinte) correspondent à ceux proposés pour l'homme adulte (Anses, 2011) car il n'existe pas de données expérimentales spécifiques. Ils sont estimés à $2 \%$ de l'apport énergétique pour le LA et à $0,8 \%$ pour l'ALA, ce qui correspond à des niveaux d'ingestion journaliers respectifs pour ces deux AGPI
Tableau 1.1. Besoins physiologiques en acides gras polyinsaturés précurseurs et à longue chaîne chez la femme enceinte ( $3^{\mathrm{e}}$ trimestre) et allaitante (Anses, 2011).

\begin{tabular}{llll}
\hline & $\begin{array}{l}\text { Acide } \\
\text { linoléique }\end{array}$ & $\begin{array}{l}\text { Acide alpha- } \\
\text { linolénique }\end{array}$ & $\begin{array}{l}\text { Acide } \\
\text { docosahexaénoïque }\end{array}$ \\
\hline Femme enceinte & $\begin{array}{l}2,0 \% \\
4,6 \mathrm{~g} / \mathrm{j}\end{array}$ & $\begin{array}{l}0,8 \% \\
1,8 \mathrm{~g} / \mathrm{j}\end{array}$ & $250 \mathrm{mg} / \mathrm{j}$ \\
Femme allaitante & $2,0 \%$ & $0,8 \%$ & $250 \mathrm{mg} / \mathrm{j}$ \\
& $5,0 \mathrm{~g} / \mathrm{j}$ & $2,0 \mathrm{~g} / \mathrm{j}$ & \\
\hline
\end{tabular}

$\%$ de l'apport énergétique sans alcool (AESA); Ration énergétique quotidienne de 8,6 MJ (femme enceinte, $2050 \mathrm{kcal}$ ) et de 9,4 MJ (femme allaitante, $2250 \mathrm{kcal}$ ) fournissant $35-40 \%$ de calories sous forme d'acides gras (ANC 2001).

Tableau 1.2. Apports nutritionnels conseillés en acides gras polyinsaturés précurseurs (ALA et LA) et à longue chaîne pour la femme enceinte ( $3^{\mathrm{e}}$ trimestre) et la femme allaitante (Anses, 2011).

\begin{tabular}{llll}
$\begin{array}{l}\text { Acide } \\
\text { linoléique }\end{array}$ & $\begin{array}{l}\text { Acide alpha- } \\
\text { linolénique }\end{array}$ & DHA & $\begin{array}{l}\text { Acides } \\
\text { gras polyinsaturés } \\
\text { longues chaînes } \\
\text { (EPA + DHA) }\end{array}$ \\
\hline
\end{tabular}

\begin{tabular}{lcccc}
\hline $\begin{array}{l}\text { Femme } \\
\text { enceinte }\end{array}$ & $4,0 \%$ & $1,0 \%$ & $250 \mathrm{mg} / \mathrm{j}$ & $500 \mathrm{mg} / \mathrm{j}$ \\
& $9,1 \mathrm{~g} / \mathrm{j}$ & $2,3 \mathrm{~g} / \mathrm{j}$ & & \\
& & & & \\
$\begin{array}{l}\text { Femme } \\
\text { allaitante }\end{array}$ & $4,0 \%$ & $1,0 \%$ & $250 \mathrm{mg} / \mathrm{j}$ & $500 \mathrm{mg} / \mathrm{j}$ \\
& $10 \mathrm{~g} / \mathrm{j}$ & $2,5 \mathrm{~g} / \mathrm{j}$ & & \\
\hline
\end{tabular}

$\%$ de l'apport énergétique sans alcool(AESA); Ration énergétique quotidienne de 8,6 MJ (femme enceinte, $2050 \mathrm{kcal}$ ) et de 9,4 MJ (femme allaitante, $2250 \mathrm{kcal}$ ) fournissant $35-40 \%$ de calories sous forme d'acides gras (ANC 2001). DHA : acide docosahexaénoïque ; EPA : acide eicosapentaénoïque.

de $5 \mathrm{~g}$ et $2 \mathrm{~g}$ pour la femme allaitante (Tab. 1.1). Les besoins physiologiques minimaux en DHA sont équivalents à ceux de l'homme adulte soit $250 \mathrm{mg} /$ jour.

Il en va de même pour les valeurs d'apports nutritionnels conseillés (Tab. 1.2) qui sont donc de $4 \%$ de l'apport énergétique pour le LA et de $1 \%$ pour l'ALA, soit 10 et $2,5 \mathrm{~g} /$ jour pour la femme allaitante. Les ANC en DHA restent les mêmes, soit $250 \mathrm{mg} /$ jour, et des ANC sont également avancés pour l'EPA + DHA (500 mg/jour).

\subsection{Apports en acides gras polyinsaturés chez la femme allaitante}

Le tableau 2 présente les résultats de l'analyse des données INCA 2, soit le profil moyen d'apports journaliers des principaux AGPI chez la femme allaitante ( $n=21 ; 23$ à 45 ans). 
Tableau 2. Apports moyens journaliers des principaux acides gras polyinsaturés de la population femmes allaitantes ( $n=21,23$ à 45 ans) au regard des recommandations.

\begin{tabular}{|c|c|c|c|c|c|c|}
\hline $\begin{array}{l}\text { Femmes allaitantes } \\
(n=21)\end{array}$ & $\begin{array}{l}\text { Besoins } \\
\text { physiologiques et } \\
\text { recommandations } \\
\text { Anses }\end{array}$ & $\begin{array}{l}\text { Apports } \\
\text { journaliers } \\
\text { moyens } \\
(\% \text { AESA })\end{array}$ & Écart-type & $\begin{array}{l}\text { Apports } \\
\text { journaliers } \\
\text { moyens }\end{array}$ & Écart-type & $\begin{array}{l}\text { Nombre de femmes } \\
\text { qui atteignent les } \\
\text { recommandations } \\
\text { Anses }\end{array}$ \\
\hline Apport énergétique total & $2250 \mathrm{kcal} / \mathrm{j}$ & & & $1911 \mathrm{kcal} / \mathrm{j}$ & 605 & $8 / 21$ \\
\hline Lipides totaux & $35-40 \%$ & 39,6 & 5,5 & $84,1 \mathrm{~g} / \mathrm{j}$ & 30 & $6 / 21$ \\
\hline LA & $\begin{array}{l}4 \%(\mathrm{ANC}) \\
2 \%(\mathrm{BP})\end{array}$ & 3,4 & 1,4 & $7,7 \mathrm{~g} / \mathrm{j}$ & 5,1 & $\begin{array}{l}5 / 21 \\
19 / 21\end{array}$ \\
\hline ALA & $\begin{array}{l}1 \%(\mathrm{ANC}) \\
0,8 \%(\mathrm{BP})\end{array}$ & 0,4 & 0,1 & $0,8 \mathrm{~g} / \mathrm{j}$ & 0,5 & $0 / 21$ \\
\hline LA/ALA & 5 & - & - & 9,4 & 2,9 & $0 / 21$ \\
\hline EPA & $250 \mathrm{mg} / \mathrm{j}$ & 0,04 & 0,02 & $82 \mathrm{mg} / \mathrm{j}$ & 51 & - \\
\hline DHA & $250 \mathrm{mg} / \mathrm{j}(\mathrm{ANC})$ & 0,05 & 0,03 & $103 \mathrm{mg} / \mathrm{j}$ & 66 & $0 / 21$ \\
\hline
\end{tabular}

AESA : apport énergétique sans alcool ; ALA : acide alpha-linolénique ; ANC : Apports nutritionnels conseillés, pour la population générale ; BP : Besoins physiologiques; DHA : acide docosahexaénoïque; EPA : acide eicosapentaénoïque ; LA : acide linoléique.

Malgré la petite taille de l'échantillon, ces données restent malgré tout intéressantes à analyser et à comparer aux recommandations actuelles de l'Anses.

Pour la population femmes allaitantes, notons tout d'abord que l'apport énergétique total (AET) moyen est bien en dessous des recommandations de l'Anses avec $1911 \mathrm{kcal}$ par jour alors même qu'un AET de $2250 \mathrm{kcal}$ par jour est recommandé. Seules 8 femmes sur 21 atteignent ces recommandations. Ce constat pourrait être lié à la surveillance accrue du poids suite à la grossesse ou au manque de temps consacré à l'alimentation en période post-partum.

En revanche, l'apport total moyen quotidien en lipides est de 39,6\% de l'apport énergétique sans alcool (AESA). Il est donc conforme aux recommandations des ANC (35-40\% AESA). Pour autant, l'analyse détaillée montre que l'apport de certains acides gras est parfois éloigné des besoins physiologiques définis par l'Anses.

L'apport moyen quotidien en LA est inférieur aux recommandations (3,4\% AESA vs. $4 \%$ recommandés) mais supérieur aux besoins physiologiques définis par l'Anses ( $2 \%$ AESA). Deux femmes sur les 21 sont néanmoins en dessous des besoins physiologiques.

Concernant les AGPI oméga-3, aucune des femmes de l'étude n'a un apport adéquat alors qu'il s'agit d'acides gras essentiels, ne pouvant être synthétisés par l'organisme. L'apport est au moins deux fois inférieur aux besoins physiologiques (BP) : 0,4\% AESA pour l'ALA (BP de 0,8\% AESA et ANC de $1 \%$ AESA), $103 \mathrm{mg}$ pour le DHA (ANC de $250 \mathrm{mg} / \mathrm{j}$ ) et $82 \mathrm{mg}$ pour l'EPA, soit $185 \mathrm{mg}$ pour EPA + DHA (ANC de $500 \mathrm{mg} / \mathrm{j}$ ). Ces apports sont même inférieurs aux apports moyens de la population générale pour ces AGPI-LC $(137 \mathrm{mg} / \mathrm{j}$ pour le DHA et $102 \mathrm{mg} / \mathrm{j}$ pour l'EPA).

Ainsi, les apports nettement insuffisants en ALA induisent un rapport LA/ALA moyen de 9,4 (pour une recommandation de l'Anses $<5$ ), malgré un apport équilibré en LA. Cette situation indique que l'alimentation des femmes allaitantes est inadaptée et ne permet pas un apport adéquat au nourrisson. La mise en place d'une campagne pédagogique à destination des femmes allaitantes et enceintes serait donc pertinente.
Il faut enfin noter que dans l'étude INCA 2, parmi les 21 femmes qui allaitent, trois ont répondu suivre un régime pour motif d'allaitement. Par ailleurs, 13 ont un apport en alcool non nul, parfois équivalent à 5 verres par semaine, bien que l'absence de consommation d'alcool soit recommandée par le corps médical.

\subsection{Contributions des groupes d'aliments chez les femmes allaitantes}

Le tableau 3 présente les trois principaux groupes d'aliments contributeurs pour chaque famille d'acides gras et pour les acides gras essentiels précurseurs ALA et LA, et n-3 à longue chaîne (DHA).

Les aliments contribuant le plus aux apports en acides gras saturés restent les graisses animales (beurre, fromages), les huiles pour les acides gras monoinsaturés et le LA, les pâtisseries et gâteaux pour l'ALA et le poisson pour le DHA.

Il est intéressant de noter que certaines catégories d'aliments qui sont consommées pendant la période d'allaitement disparaissent du podium spécifiquement au cours de la période de grossesse pour des raisons de sécurité nutritionnelle (résultats non montrés). C'est le cas des fromages pour lesquels il existe des conseils d'éviction pour des risques de listériose, mais également des crustacés et des mollusques dont la consommation est déconseillée (listériose et salmonellose). Ainsi la contribution en DHA de ces derniers devient négligeable pendant la grossesse.

Enfin, la contribution des poissons à l'apport en DHA, qui est basse en période d'allaitement, chute brutalement pendant la grossesse. Cela peut s'expliquer par la combinaison de deux facteurs :

- les conseils de limitation de consommation des poissons gras pour cause de risque de contamination en métaux lourds;

- les conseils d'éviction des poissons crus pour cause de risque de listériose, alors même qu'ils sont de plus en plus fréquemment consommés par la population française (Anses, 2017). 
Tableau 3. Les trois principaux groupes d'aliments contributeurs pour chaque famille d'acide gras chez les femmes allaitantes $(n=21$; 23-45 ans).

\begin{tabular}{ll}
\hline & Femmes allaitantes \\
\hline Lipides totaux & Beurre $(14,8 \%)$ \\
& Huile $(11 \%)$ \\
& Fromages $(9,2 \%)$ \\
AGS & Beurre $(23,6 \%)$ \\
& Fromages $(14 \%)$ \\
& Viande $(7,8 \%)$ \\
AGMI & Huile $(17,4 \%)$ \\
& Beurre $(10 \%)$ \\
AGPI & Viande $(9,3 \%)$ \\
& Huile $(18,3 \%)$ \\
LA & Condiments et sauces $(9,1 \%)$ \\
& Plats composés $(8,8 \%)$ \\
& Huile $(24,5 \%)$ \\
ALA & Plats composes $(9,9 \%)$ \\
& Condiments et sauces $(8 \%)$ \\
& Pâtisseries et gâteaux $(10,8 \%)$ \\
DHA & Huile $(10,3 \%)$ \\
& Plats composes $(9,6 \%)$ \\
& Poissons $(68 \%)$ \\
& Crustacés et mollusques $(13 \%)$ \\
& Eufs et dérivés $(10 \%)$ \\
\hline
\end{tabular}

Les contributions sont données en pourcentage de l'AESA (apport énergétique sans alcool). ALA: acide alpha-linolénique; AGS: acides gras saturés; AGMI: acides gras monoinsaturés; AGPI: acides gras polyinsaturés; LA: acide linoléique; DHA: acide docosahexaénoïque.

À l'inverse, la catégorie Plats composés est plus représentée pour les apports en AGPI (ALA, LA et DHA). Cela illustre le changement de comportement alimentaire lié à la réduction du temps dédié à la cuisine à l'arrivée d'un nourrisson.

\section{3 Évolution du contenu en acides gras essentiels du lait maternel de 1997 à 2014}

\subsection{Méthodologie}

Trois études nutritionnelles indépendantes (Vaysse et al., 2011; Mazurier et al, 2017) ont été conduites entre 1997 et 2014 chez des mères allaitantes dans 4 à 8 lactariums de France. L'objectif secondaire de ces études épidémiologiques consistait à évaluer les niveaux de consommation des femmes allaitantes françaises en acides gras oméga-6 et oméga-3 pendant la période périnatale.

Au total, 238 femmes âgées de 19 à 39 ans, à un stade compris entre 37 et 40 semaines de gestation, ont été recrutées en 1997 $(n=16), 2007(n=142)$ et $2014(n=80)$. Les participantes de l'étude ont été informées des objectifs avant recueil de leur consentement écrit. Les protocoles des différentes études ont été approuvés par le Comité d'éthique de Bordeaux.

Pour l'ensemble des études, $15 \mathrm{ml}$ de lait maternel mature ont été collectés au matin, pendant les premières tétées, entre 1 et 3 mois post-partum. La composition en acides gras des échantillons de lait maternel a été déterminée par chromatographie en phase gazeuse haute performance, la quantité de chaque acide gras étant exprimée en pourcentage des acides gras totaux (Boué et al., 2000).

La comparaison des données issues de ces 3 études a été faite par le test statistique non paramétrique de Kruskall et Wallis (différences significatives au seuil de $p \leq 0,05$ ).

\section{2 Étude comparative du statut nutritionnel du lait maternel comme reflet de l'évolution de la consommation lipidique en France}

La composition en acides gras du lait maternel est un marqueur reconnu de la consommation des AGPI alimentaires. Dans ce cadre, le suivi de l'évolution de cette composition permet d'estimer l'évolution de la consommation des AGPI sur une population spécifique de mères allaitantes, en France.

Le tableau 4 rapporte les proportions moyennes en AGPI du lait mature de la femme allaitante française acquises en 1997, 2007 et 2014.

Les AGPI constituent en moyenne $13 \%$ à $15 \%$ des acides gras totaux du lait (AG totaux); près de $90 \%$ de ces AGPI étant des AGPI oméga- 6 et les $10 \%$ restants étant des AGPI oméga-3. Plus précisément, les AGPI oméga-6 sont majoritairement représentés par le LA (en moyenne $10 \%$ à $13 \%$ des AG totaux, soit près de $87 \%$ à $96 \%$ des AGPI oméga-6 totaux) et en proportion moindre par l'AA (en moyenne, $0,4 \%$ des AG totaux, soit $3 \%$ des AGPI oméga- 6 totaux).

Pour les AGPI oméga-3, c'est le précurseur, l'ALA qui prédomine (en moyenne $0,5 \%$ à $1 \%$ des AG totaux, soit $47 \%$ à $69 \%$ des AGPI oméga-3 totaux), suivi du DHA $(0,2-0,3 \%$ des AG totaux). Enfin, le rapport LA/ALA varie de 12 à 28 selon l'année considérée.

La comparaison de l'ensemble des données a mis en évidence une évolution favorable des contenus en AGPI oméga3 et oméga-6 du lait maternel entre 1997 et 2007 , qui se confirme en 2014. Ainsi, le taux de LA a diminué de $25 \%$ (passant de $13,3 \%$ en 1997 à $10,0 \%$ des AG totaux en 2014) alors que celui en ALA a été doublé (passant de 0,5\% en 1997 à 1,0\% des AG totaux en 2014). Ainsi, le rapport LA/ALA qui était égal à 28 en 1997 est passé à 12 en 2014, améliorant ainsi la valeur de ce ratio au regard des recommandations nutritionnelles pour le nouveauné (ratio préconisé de 6 [Anses, 2011]). Les contenus en AA et DHA n'ont pas évolué significativement au cours de la période considérée.

Ces observations résultent vraisemblablement d'une évolution positive des habitudes alimentaires françaises, caractérisées par un «rééquilibrage» favorable des apports en précurseurs oméga- 6 et oméga- 3 au cours de ces 2 dernières décennies. Néanmoins, force est de constater que malgré cette évolution, il n'en reste pas moins que les apports en ALA et DHA de la population française sont encore loin des recommandations nutritionnelles.

\subsection{Effet d'une supplémentation nutritionnelle en acides gras polyinsaturés oméga-3 sur la composition du lait maternel de femmes allaitantes}

L'objectif de l'étude menée en 2014 sur 80 femmes allaitant leur bébé consistait à évaluer les bénéfices d'un apport 
Tableau 4. Profil moyen de composition en acides gras polyinsaturés oméga-3 et oméga- 6 ( $\%$ des acides gras totaux) du lait mature de la femme allaitante française entre 1997 et 2014.

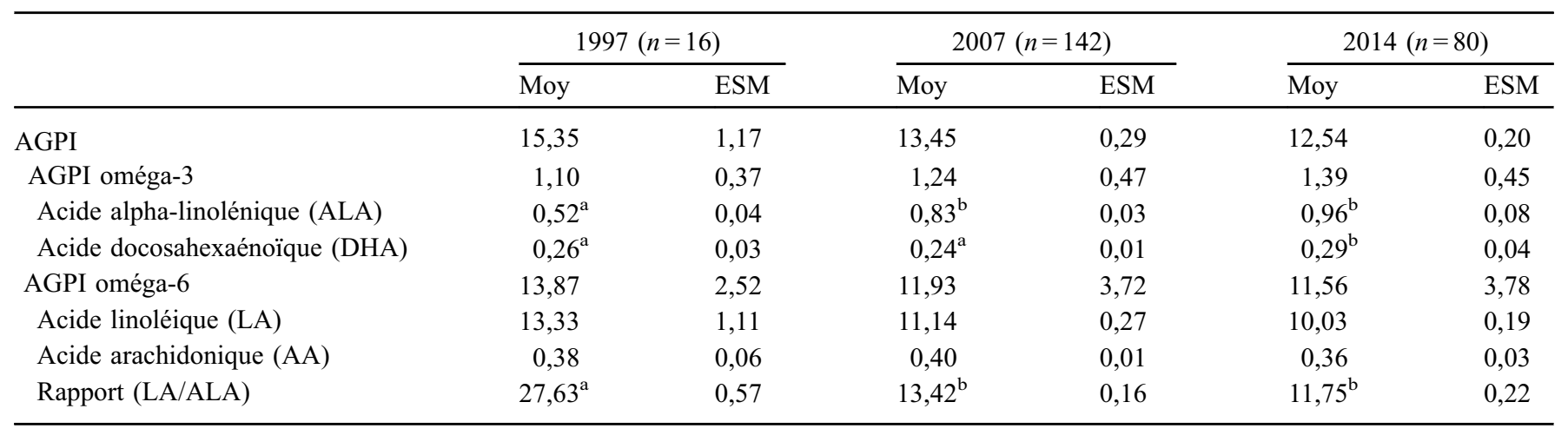

AGPI : acides gras polyinsaturés; Moy: moyenne; ESM : erreur standard à la moyenne. Les moyennes marquées de lettres différentes sont significativement différentes $(p<0,05)$, test statistique non paramétrique de Kruskall et Wallis.

Tableau 5. Apports quotidiens en lipides et en acide alpha-linolénique (g/j) apportés par les 4 régimes de l'étude de supplémentation en acides gras polyinsaturés oméga-3 chez la femme allaitante.

\begin{tabular}{lcccc}
\hline Régimes & Olive $(\mathrm{T})$ & Margarine ALA $(\mathrm{M})$ & Huile colza $(\mathrm{H})$ & Huile de colza + Margarine ALA $(\mathrm{MH})$ \\
\hline Apport en lipides $(\mathrm{g} / \mathrm{j})$ & 46 & 46 & 46 & 46 \\
Apport en ALA $(\mathrm{g} / \mathrm{j})$ & 0,3 & 1,4 & 3,2 & 4,3 \\
Ratio LA/ALA & 8,3 & 5,3 & 2 & 2,6 \\
\hline
\end{tabular}

ALA : acide alpha-linolénique; LA : acide linoléique.

Tableau 6. Composition en acides gras polyinsaturés oméga-3 (\% des acides gras totaux) du lait maternel au terme des 4 régimes de l'étude de supplémentation en acides gras polyinsaturés oméga-3 chez la femme allaitante.

\begin{tabular}{|c|c|c|c|c|c|c|c|c|}
\hline & \multicolumn{2}{|c|}{ Olive (T) $n=22$} & \multicolumn{2}{|c|}{ Margarine ALA (M) } & \multicolumn{2}{|c|}{ Huile colza $(\mathrm{H})$} & \multicolumn{2}{|c|}{ Huile de colza + Margarine ALA $(\mathrm{MH})$} \\
\hline ALA & 0,77 & 0,06 & $1,09^{*}$ & 0,14 & $1,29 *$ & 0,22 & $2,15^{*}$ & 0,55 \\
\hline DHA & 0,39 & 0,03 & 0,42 & 0,06 & 0,42 & 0,03 & 0,54 & 0,16 \\
\hline Ratio LA/ALA & 11,18 & 7,06 & $8,95^{*}$ & 12,85 & $8,99 *$ & 11,06 & $5,54 *$ & 4,23 \\
\hline
\end{tabular}

Les moyennes marquées d'un astérisque sont significativement différentes $(p<0,05)$ du groupe Témoin.

en ALA (apporté sous forme d'huile de colza et/ou de margarine riche en ALA) sur le contenu en AGPI oméga-3 du lait maternel, afin de définir « le type » d'alimentation lipidique le mieux adapté à la femme allaitante et à son nouveau-né.

Cette étude a consisté en une intervention nutritionnelle multicentrique (Bordeaux, Lyon, Paris et Montpellier), randomisée selon un plan factoriel en 4 groupes parallèles (de 20 femmes chacun) supplémentés en ALA pendant 15 jours (l'ALA étant apporté sous forme d'huile de colza ou de margarine riche en ALA, ou de margarine riche en ALA + huile de colza) ou non supplémentés en ALA (groupe témoin consommant de l'huile d'olive) (Tab. 5). L'intervention nutritionnelle a été précédée d'une période de «wash-out» en ALA de 15 jours durant laquelle les femmes ont consommé un régime à base d'huile d'olive.

En outre, toutes les femmes de l'étude ont également consommé 150 grammes de maquereau deux fois par semaine de façon à «standardiser» le niveau d'apport en AGPI-LC oméga-3 entre les différents groupes.
Le profil en acides gras du lait maternel a été déterminé pour chaque groupe, par l'ITERG, au terme de l'intervention nutritionnelle.

Les résultats (Tab. 6) montrent une augmentation significative du contenu en ALA du lait maternel suite à la consommation journalière d'huile de colza et/ou de margarine riche en ALA comparativement à une diète n'apportant pas d'ALA, à base d'huile d'olive (respectivement, $+42 \%$ groupe $\mathrm{M},+68 \%$ groupe $\mathrm{H}$ et $+179 \%$ groupe $\mathrm{MH})$. De plus, la concentration en ALA du lait maternel des femmes supplémentées en ALA est en accord avec les apports recommandés pour le nouveau-né c'est-à-dire $1 \%$ à $4 \%$ des $A G$ totaux (Agostoni et al., 2010; Innis, 2007b; Koletzko et al., 2008; Lapillonne, 2013). On notera que la proportion d'ALA dans le lait des mères ayant consommés $3,2 \mathrm{~g}$ d'ALA $/ \mathrm{j}$ sous forme $\mathrm{d}$ 'huile de colza est comparable à celle observée dans le lait des mères ayant consommé 2 fois moins d'ALA (1,4 g d'ALA/j) sous forme de margarine riche en ALA, suggérant un impact de la forme d'apport de l'ALA sur sa teneur dans le lait maternel. 
Comme attendu, une consommation plus importante d'ALA $(4,3 \mathrm{~g} / \mathrm{j})$ avec l'apport combiné d'huile de colza et de margarine riche en oméga-3 conduit à des teneurs en ALA du lait maternel significativement plus élevées qu'avec des apports moindres en ALA sous forme d'une seule source d'ALA (huile de colza ou margarine riche en ALA). Cependant, l'enrichissement en ALA des laits maternels des groupes M, H et MH ne s'accompagne pas d'une augmentation significative de la proportion de DHA dans ces mêmes laits maternels (comparativement au groupe témoin non supplémenté) mais répond néanmoins aux apports en DHA recommandés pour le nouveau-né $(0,32 \%$ des AG totaux). La teneur en DHA des laits maternels est comparable entre les différents groupes, vraisemblablement du fait de l'apport en DHA identique entre les groupes (soit l'équivalent de $350 \mathrm{mg} / \mathrm{j}$ de DHA fournis par la consommation de maquereau vs. les $250 \mathrm{mg} / \mathrm{j}$ de DHA recommandés pour les femmes adultes [Anses, 2011]). Ce résultat est cohérent avec les études d'intervention précédentes indiquant que l'apport alimentaire en DHA de la mère est le facteur qui impacte le plus la teneur en DHA du lait maternel (Innis, 2007a).

Néanmoins, on notera une teneur en DHA dans le lait maternel du groupe $\mathrm{MH}$ qui tendrait à être plus importante que chez les autres groupes $\mathrm{M}$ ou $\mathrm{H}$, suggérant que le niveau d'apport en ALA dans ce groupe (4,3 g/j) semble suffisamment élevé pour «compléter»l'enrichissement en DHA du lait maternel, induit par la consommation de maquereau. Cette hypothèse pouvant être confortée par le fait que le rapport LA/ ALA observé dans le lait maternel du groupe MH (LA/ $\mathrm{ALA}=5,5)$ soit le plus favorable à la bioconversion de l'ALA en DHA (Ares Segura et al., 2016). Ces données mériteraient d'être vérifiées sur un nombre plus conséquent de mères allaitantes dans une nouvelle étude.

Les résultats de cette étude de supplémentation en AGPI oméga-3 confortent la recommandation d'un régime équilibré et varié pour les femmes allaitantes. Ce régime devrait inclure les poissons gras en raison de leur teneur en DHA ainsi que la margarine enrichie en oméga-3 et l'huile de colza pour obtenir le rapport LA/ALA le plus favorable $(5,5)$ pour la synthèse du DHA chez le nourrisson. Des études complémentaires sont nécessaires pour définir les niveaux d'apport en oméga-3 (tant l'ALA que le DHA) spécifique à la période périnatale et conduire potentiellement à la formulation de produits alimentaires destinées aux mères allaitantes et aidant à atteindre une concentration élevée en DHA dans le lait maternel.

Cette étude de supplémentation nutritionnelle en acides gras polyinsaturés oméga-3 a été réalisée avec le $\mathrm{CHU}$ de Bordeaux (CIC Pédiatrique, Unité de Soutien Méthodologique à la Recherche Clinique et Épidémiologique) et les Equipes des lactariums de Bordeaux-Marmande, Lyon, Montpellier et Paris en collaboration avec les sociétés Lesieur et St Hubert, et a bénéficié d'un financement de type "Programme Hospitalier de Recherche Clinique» pour couvrir les coûts relatifs au recrutement des femmes allaitantes, ainsi que d'un financement de Terres Univia pour l'analyse des lipides du lait maternel.

\section{Conclusions}

Bien que les résultats de l'étude de consommation en acide gras polyinsaturés soient basés sur un échantillon réduit de femmes et qu'il s'agisse de relevés de consommation datant des années 2006-2007, ces données traduisent des tendances intéressantes à analyser. Les comparaisons avec les données INCA 3 (Anses, 2017) resteront néanmoins limitées comptetenu du changement de méthodologie opéré par l'Anses.

Les études de suivi de l'évolution du contenu en acides gras essentiels du lait maternel montrent une évolution positive du comportement alimentaire en France. Les apports en ALA et DHA de la population française restent néanmoins en deçà des recommandations nutritionnelles. L'étude de supplémentation apporte également un éclairage sur l'intérêt de privilégier chez les femmes allaitantes, la consommation de sources lipidiques naturellement riches en ALA et /ou DHA pour améliorer le contenu en oméga-3 du lait maternel. Des études complémentaires sont nécessaires pour définir les niveaux d'apport en oméga-3 (tant l'ALA que le DHA) spécifique à la période périnatale.

L'ensemble de ces données permettent de conclure qu'il faut améliorer l'éducation nutritionnelle de la population des femmes en situation d'allaitement, en adaptant les messages de santé publique spécifiques. Il est également nécessaire de rééquilibrer l'alimentation de ces femmes en privilégiant les sources végétales d'AGPI n-3 (huile de colza, de noix, margarines riches en ALA), en parallèle des apports liés aux poissons gras. Le développement d'une offre alimentaire en plats préparés formulés pour les besoins des femmes en situation d'allaitement pourrait être une piste d'amélioration de la situation actuelle, et ce d'autant plus que cela serait bénéfique à toute la population.

Enfin, un projet d'observatoire du lait maternel est en cours de construction par Terres Univia, le CNIEL et l'ITERG. Il permettra de suivre l'évolution des habitudes de consommation en acides gras des femmes allaitantes françaises à travers l'analyse de la composition en acides gras du lait maternel, marqueur reconnu de la consommation en acides gras notamment polyinsaturés.

Remerciements. Terres Univia et l'ITERG remercient le GLN d'avoir permis la présentation de ces travaux lors de la journée GLN du 21 novembre 2017.

Les auteurs tiennent à remercier les femmes allaitantes pour leur participation aux différentes études, ainsi que les équipes des lactariums, en particulier Dr Delphine Lamireau (Lactarium Bordeaux-Marmande), Pr Jean-Charles Picaud \& Rachel Buffin (Lactarium de Lyon), Dr Evelyne Mazurier et Dr Gilles Cambonie (Lactarium de Montpellier), Dr Virginie Rigourd (Lactarium Necker Enfants Malades), Pr Chantal Maurage, (Lactarium de Tours), Pr Frédérique Huet (Lactarium de Dijon), Dr Cécile Boscher (Lactarium de Nantes), Dr Virginie Rigourd (Lactarium d'Île-de- France), Pr Olivier Garraud (Lactarium de St Étienne) pour leur collaboration, Fabienne Nacka et Wafae Belcadi (CIC Pédiatrique 1401, CHU Pellegrin, Bordeaux) pour la coordination des études 2007 et 2014, Paul Perez et Rémi Sitta (Unité de Soutien Méthodologique à la Recherche Clinique et Épidémiologique, CHU de Bordeaux) pour l'exploitation statistique des données de l'étude de supplémentation en oméga-3, les sociétés Lesieur et St Hubert pour avoir fourni les huiles et margarines, lors de l'étude de supplémentation en oméga-3, et Laurence Fonseca, Sandrine Djoukitch et Émeline Montesinos (ITERG) pour l'analyse des échantillons de lait maternels. 
Ces études ont été réalisées avec le soutien financier de FranceAgriMer (Établissement national des produits de l'agriculture et de la mer) et de Terres Univia.

Conflits d'intérêts. Noëmie Simon est salariée de Terres Univia.

Jessica Tressou, Stéphane Pasteau et Philippe Guesnet collaborent avec Terres Univia pour l'analyse des données INCA 2.

Benjamin Buaud, Leslie Coüedelo et Carole Vaysse sont salariés de l'ITERG.

Claude Billeaud est salarié du CIC pédiatrique $1401 \mathrm{du}$ CHU Pellegrin de Bordeaux

\section{Références}

Agostoni C, Buonocore G, Carnielli VP, et al. 2010. Enteral nutrient supply for preterm infants: Commentary from the European Society of Paediatric Gastroenterology, Hepatology and Nutrition Committee on Nutrition. J Pediatr Gastroenterol Nutr 50: 85-91. DOI: 10.1097/MPG.0b013e3181adaee0.

Anses. 2009. Étude Individuelle Nationale des Consommations Alimentaires 2 (INCA 2) (2006-2007). Rapport d'expertise collective. Disponible sur https://www.anses.fr/fr/system/files/ PASER-Ra-INCA2.pdf.

Anses. 2011. Actualisation des apports nutritionnels conseillés pour les acides gras. Rapport d'expertise collective. Disponible sur https://www.anses.fr/fr/system/files/NUT2006sa0359Ra.pdf.

Anses. 2013. Table de composition nutritionnelle des aliments Ciqual. Disponible sur https://pro.anses.fr/TableCIQUAL/index.htm.

Anses. 2015. Apports en acides gras de la population vivant en France et comparaison aux apports nutritionnels conseillés définis en 2010. Disponible sur https:/www.anses.fr/fr/system/files/ NUT2014sa0117Ra.pdf.

Anses. 2017. Étude individuelle nationale des consommations alimentaires 3 (INCA 3) (2014-2015). Rapport d'expertise collective. Disponible sur https://www.anses.fr/fr/system/files/NUT2014 SA0234Ra.pdf.

Ares Segura S, Arena Ansótegui J, Díaz-Gómez NM, En Representación del Comité de Lactancia Materna de la Asociación Espanola de Pediatría. 2016. La importancia de la nutrición materna durante la lactancia: ¿Necesitan las madres lactantes suplementos nutricionales? [The importance of maternal nutrition during breastfeeding: Do breastfeeding mothers need nutritional supplements?]. An Pediatr 84(6): 347.e7.

Boué C, Combe N, Billeaud C, et al. 2000. Trans fatty acids in adipose tissue of French women in relation to their dietary sources. Lipids 35: 561-566.

Buaud B, Tressou B, Guesnet P, Simon N, Pasteau P. 2018. Inadequacy of n-3 polyunsaturated fatty acid dietary intakes in the general French population of elderly (65 to 79 years old): the
INCA 2 survey. J Aging Res Clin Practice 7: 69-74. Available from https://doi.org/10.14283/jarcp.2018.13.

Guesnet P, Alessandri JM, Astorg P, Pifferi F, Lavialle M. 2005. Les rôles physiologiques majeurs exercés par les acides gras polyinsaturés (AGPI). OCL 12(5-6): 333-343.

Innis SM. 2004 Polyunsaturated fatty acids in human milk: an essential role in infant development. Adv Exp Med Biol 554: $27-43$.

Innis SM. 2007a. Human milk: maternal dietary lipids and infant development. Proc Nutr Soc 66(3): 397-404.

Innis SM. 2007b. Dietary (n-3) fatty acids and brain development. $J$ Nutrition 137(4): 855-859.

Jensen RG. 1999. Lipids in human milk. Lipids 34: 1243-71.

Koletzko B, Thiel I, Abiodun PO. 1992. The fatty acid composition of human milk in Europe and Africa. J Pediatr 120: S62-70.

Koletzko B, Lien E, Agostoni C, et al. 2008. The roles of long-chain polyunsaturated fatty acids in pregnancy, lactation and infancy: Review of current knowledge and consensus recommendations. $J$ Perinat Med 36(1): 5-14.

Lapillonne A, Groh-Wargo S, Gonzalez CH, Uauy R. 2013. Lipid needs of preterm infants: updated recommendations. Journal of Pediatrics 162(3): S37-S47.

Lauritzen L, Jorgensen MH, Mikkelsen TB, et al. 2004. Maternal fish oil supplementation in lactation: effect on visual acuity and n-3 fatty acid content of infant erythrocytes. Lipids 39: 195-206.

Mazurier E, Rigourd V, Perez P, et al. 2017. Effects of maternal supplementation with Omega-3 precursors on human milk composition. J Hum Lact 33(2): 319-328. DOI: 10.1177/ 0890334417691946.

Read WW, Lutz PG, Tashjian A. 1965. Human milk lipids. II. The influence of dietary carbohydrates and fat on the fatty acids of mature milk. A study in four ethnic groups. Am J Clin Nutr 17: $180-183$

Simopoulos AP. 2011 Importance of the omega-6/omega-3 balance in health and disease: evolutionary aspects of diet. World Rev Nutr Diet 102: 10-21

Sioen I, van Lieshout L, Eilander A, et al. 2017. Systematic review on $\mathrm{N}-3$ and N-6 polyunsaturated fatty acid intake in European countries in light of the Current Recommendations- Focus on specific population groups. Ann Nutr Metab 70(1): 39-50

Tressou J, Pasteau S, Darrigo Dartinet S, Simon N, Le Guillou C. 2016. New data about dietary intake of fatty acids in the French population. OCL 23(3): D303.

Uauy R, Hoffman DR, Mena P, Llanos A, Birch EE. 2003. Term infant studies of DHA and ARA supplementation on neurodevelopment: results of randomized controlled trials. J Pediatr 143: S17-25.

Vaysse C, Billeaud C, Guesnet P, et al. 2011. Teneurs en acides gras polyinsaturés essentiels du lait maternel en France : évolution des teneurs en acides linoléique et alpha-linolénique. Med Nutr 47(2): 5-9. Disponible sur https://doi.org/10.1051/mnut/201120003.

Citation de l'article : Vaysse C, Simon N, Tressou J, Pasteau S, Buaud B, Guesnet P, Couedelo L, Billeaud C. 2018. Niveau de consommation en acides gras polyinsaturés de la femme allaitante en France : étude de consommation INCA2 et évolution du contenu en acides gras essentiels du lait maternel de 1997 à 2014. OCL 25(3): D304. 